\title{
In vivo Molecular targeting effects of anti-Sp17- ICG-Der-02 on hepatocellular carcinoma evaluated by an optical imaging system
}

\author{
Fang-qiu Li $i^{*}$, Shi-xin Zhang ${ }^{1}$, Lian-xiao $\mathrm{An}^{2}$, Yue-qing $\mathrm{Gu}^{2^{*}}$
}

\begin{abstract}
Background: As the expression of human sperm protein 17 (Sp17) in normal tissue is limited and the function is obscure, its aberrant expression in malignant tumors makes it to be a candidated molecular marker for tumor imaging diagnosis and targeting therapy of the diseases.The aim of this research is to evaluate the targeting effects of anti-sperm protein 17 monoclonal antibody (anti-Sp17) on cancer in vivo and investigate its usefulness as a reagent for molecular imaging diagnosis.

Methods: Immunohistochemistry was used to identify the expression of Sp17 in a hepatocellular carcinoma cell line and tumor xenograft specimens. A near infrared fluorescence dye, ICG-Der-02, was covalently linked to antiSp17 for in vivo imaging. The immuno-activity of the anti-Sp17-ICG-Der-02 complex was tested in vitro by ELISA; it was then injected into tumor-bearing nude mice through the caudal vein to evaluate its tumor targeting effect by near infrared imaging system.
\end{abstract}

Results: Overexpression of Sp17 on the surface of the hepatocellular carcinoma cell line SMMC-7721 was demonstrated. Anti-Sp17-ICG-Der-02 with immuno-activity was successfully synthesized. The immuno-activity and photo stability of anti-Sp17- ICG-Der-02 showed good targeting capability for Sp17 expressing tumor models (SMMC-7721) in vivo, and its accumulation in the tumor lasted for at least 7 days.

Conclusions: Anti-Sp17 antibody targeted and accumulated in Sp17 positive tumors in vivo, which demonstrated its capability of serving as a diagnostic reagent.

\section{Introduction}

Cancer remains one of the leading causes of death in the world. Despite advances in our understanding of molecular and cancer biology, the discovery of cancer biomarkers and the refinement of conventional surgical procedures, radiotherapy, and chemotherapy, the overall survival rate from cancer has not significantly improved in the past two decades [1,2]. Early noninvasive detection and characterization of solid tumors is a fundamental prerequisite for effective therapeutic intervention. Emerging molecular imaging techniques now allow

\footnotetext{
* Correspondence: njlifq@163.com; cupyueqing@163.com

'Laboratory of Molecular Biology, Institute of Medical Laboratory Sciences, Jinling Hospital, School of Medicine, Nanjing University, Nanjing 210002, China

${ }^{2}$ Department of Biomedical Engineering, School of Life Science and Technology, China Pharmaceutical University, Nanjing, 210009, China Full list of author information is available at the end of the article
}

recognition of early biomarker and anatomical changes before manifestation of gross pathological changes [3-6]. The development of novel approaches for in vivo imaging and personalized treatment of cancers is urgently needed to find cancer-specific markers, but there is still limited knowledge of suitable biomarkers.

Sperm protein 17 (Sp17) was originally reported to be expressed exclusively in the testis. Its primary function is binding to the zona pellucida and playing a critical role in successful fertilization [7]. Expression of Sp17 in malignant cells was first described by Dong et al, who found the mouse homologue of Sp17 to be highly expressed in metastatic cell lines derived from a murine model of squamous cell carcinoma but not in the nonmetastatic parental line [8]. Various researchers have demonstrated the aberrant expression of Sp17 in malignant tumors including myeloma [9], primary ovarian tumors $[10,11]$, neuroectodermal and meningeal

\section{Biomed Central}


tumors [12], and esophageal squamous cell cancers [13]. Sp17 was found in $66 \%$ of endometrial cancers (11), and $61 \%$ of cervical cancers [14] in our previous work. As the expression of $\mathrm{Sp} 17$ in normal tissue is limited and its function is obscure, it is reasonable to predict that aberrant expression of Sp17 in malignant tumors could be a molecular marker for tumor imaging diagnosis and targeting therapy of the diseases.

Molecular imaging methods permit noninvasive detection of cellular and molecular events by using highly specific probes and gene reporters in living animals, some of which can be directly translated to patient studies. A novel optical imaging technique in cancer is the use of near-infrared (NIR) light (700 to $900 \mathrm{~nm}$ ) to monitor the site and size of the cancers [15]. The fundamental advantage of imaging in the NIR range is that photon penetration into living tissue is higher because of lower photon absorption and scatter [16]. An additional advantage is that tissue emits limited intrinsic fluorescence (i.e., autofluorescence) in the $700 \mathrm{~nm}$ to $900 \mathrm{~nm}$ range. Therefore, fluorescence contrast agents that emit in the NIR range demonstrate a favorable signal-to-background ratio(SBR) when used in animal models or for patient care, especially for endoscopy. Optical imaging is a very versatile, sensitive, and powerful tool for molecular imaging in small animals.

The near infrared fluorescence dye ICG-Der-02 (indocyanine Green derivative 02) is a derivative of indocyanine green (ICG), which was approved by the FDA (Food and Drug Administration) to be used in human subjects. Compared to ICG, the self-synthesized ICGDer-02 organic dye holds favorable hydrophilicity and higher fluorescence quantum yield with excitation and emission peaks at $780 \mathrm{~nm}$ and $810 \mathrm{~nm}$, respectively. ICG-Der-02 offers one carboxyl functional group on the side chain which enables the dye to be covalently conjugated to the biomarker for in vivo optical imaging [17].

In this study, we first demonstrated the overexpression of Sp17 in the hepatocellular carcinoma cell line SMMC-7721 and in xenografts in mice. After synthesis of anti-Sp17-ICG-Der-02, we evaluated the targeting effect of anti-Sp17-ICG-Der-02 on tumors in vivo with a whole-body optical imaging system in animal models.

\section{Materials and methods}

\section{Cell line and monoclonal antibody}

The human hepatocellular carcinoma cell line SMMC7721 expresses high levels of Sp17 and was used for in vitro and in vivo experiments, Sp17- HO8910 ovarian cancer cell line used as negative control. The cells were cultured in RPMI 1640 medium (Invitrogen) supplemented with $10 \%$ fetal bovine serum (Hyclone) in a humidified incubator maintained at $37^{\circ} \mathrm{C}$ with $5 \% \mathrm{CO}_{2}$ atmosphere and medium was replaced every 3 days. The anti-human Sp17 monoclonal antibody clone 3C12 was produced in our laboratory as previously described [14]. Monoclonal antibodies were purified from hybridoma ascites using a HiTrap Protein G HP affinity column (Amersham Biosciences).

\section{Tumor animal models}

Male athymic nude mice (6-8 wk old, 18-22 g) were housed in a pathogen-free mouse colony and provided with sterilized pellet chow and sterilized water. All experiments were performed in accordance with the guidelines of the Animal Care Committee of the hospital. SMMC-7721 cells were treated with trypsin when near confluence and harvested. Cells were pelleted by centrifugation at $1200 \mathrm{rpm}$ for $5 \mathrm{~min}$ and resuspended in sterile culture medium, then implanted subcutaneously into the flank of the mice $\left(2 \times 10^{6}\right.$ cells per animal). The mice were subjected to optical imaging studies when the tumor volume reached $0.5 \sim 1.8 \mathrm{~cm}$ in diameter.

\section{Immunocytochemical and immunohistochemical analysis}

To investigate the expression of Sp17 in the SMMC7721 and HO8910 cell lines, cells were cultured on a coverglass and then fixed with cooled acetone. AntiSp17 monoclonal antibody was then added at a concentration of $2 \mu \mathrm{g} / \mathrm{ml}$ and incubated overnight at $4^{\circ} \mathrm{C}$. The primary antibody was detected with anti-mouse IgG labeled with horseradish peroxidase (DAKO). Diaminobenzidine (DAB) substrate was added for 7 min followed by washing with deionized water and hematoxylin was applied for $1 \mathrm{~min}$ to counterstain the cell on slices. Then the cell slices were dehydrated via graded ethanols followed by xylene and coverslips were attached with permount. The immunocytochemical reaction turned brown and was observed using a light microscope.

Tumor tissue sections $(3 \mu \mathrm{m})$ from mouse model were placed on glass slides, heated at $60^{\circ} \mathrm{C}$ for $20 \mathrm{~min}$, and then deparaffinized with xylene and ethanol. For antigen retrieval, tumor specimens mounted on glass slides were immersed in preheated antigen retrieval solution (DAKO high $\mathrm{pH}$ solution; DAKO) for $20 \mathrm{~min}$ and cooled for $20 \mathrm{~min}$ at room temperature. After the inactivation of endogenous peroxidase, the tissue slices were treated with anti-Sp17 monoclonal antibody and unrelated monoclonal antibody (mose anti-Candida enolase) with the same protocol as immunocytochemistry.

\section{Synthesis of anti-Sp17-ICG-Der-02}

The synthesis of the anti-Sp17-ICG-Der-02 complex was conducted in three consecutive steps: First, the dye $(1 \mathrm{mg}$, $0.001 \mathrm{mmol})$ was dissolved in $\mathrm{H}_{2} \mathrm{O}(0.5 \mathrm{ml})$ and mixed with the catalysts EDC $(2.90 \mathrm{mg}, 0.015 \mathrm{mmol})$ and NHS (1.73 mg, $0.015 \mathrm{mmol}$ ) (GL Biochem Co. Ltd, Shanghai, 
China) for the activation of the carboxylic acid functional group for about $4 \mathrm{~h}$ at room temperature. Next, the active ICG-Der-02 solution was added dropwise to $50 \mu \mathrm{l}$ $(200 \mu \mathrm{g})$ anti-Sp17 solution and then stirred at $4{ }^{\circ} \mathrm{C}$ for $10 \mathrm{~h}$ in the dark. The reaction was quenched by adding $200 \mu \mathrm{l}$ of $5 \%$ acetic acid (HOAc). Finally, the mixture was dialyzed (molecular weight cutoff $10 \mathrm{kDa}$ ) against $0.1 \mathrm{~mol} /$ L phosphate buffer solutions $(\mathrm{pH}=8.3)$ until no free dye dialyzed out. The absorption and fluorescence emission peaks of anti-Sp17-ICG-Der-02 were located at $780 \mathrm{~nm}$ and $835 \mathrm{~nm}$, which is exact the same as the pure ICGDer-02, indicating the conjugation had no effect on the optical properties of NIR dye. The purified Sp17-ICGDer-02 conjugates were stored at $4^{\circ} \mathrm{C}$ in the dark for future use.

\section{ELISA for immunological activity of ICG-Der-02 labeled anti-Sp17}

Recombinant human sperm protein 17 produced in our laboratory [14] at $1 \mu \mathrm{g} / \mathrm{ml}$ in coating buffer were added to 96 -well plates $(100 \mu \mathrm{l} /$ well $)$ and incubated overnight at $4{ }^{\circ} \mathrm{C}$. The plates were then washed with $0.05 \%$ Tween 20/PBS and blocked with $100 \mu \mathrm{l} /$ well of $5 \%$ fetal calf serum/PBS for $1 \mathrm{~h}$ at $37^{\circ} \mathrm{C}$. After washing, ICG-Der-02 labeled or naked anti-Sp17 (100 $\mu \mathrm{l} /$ well), serially diluted with $5 \%$ fetal calf serum/PBS, was added and the plates were incubated for $1 \mathrm{~h}$ at $37^{\circ} \mathrm{C}$. After a third washing, 1:2000 diluted goat anti-mouse IgG labeled with horseradish peroxidase $(100 \mu \mathrm{l} /$ well $)$ was added and the plates were incubated for $1 \mathrm{~h}$ at $37^{\circ} \mathrm{C}$. After another washing substrate TMB solution was added to each well and the plates were incubated for $10 \mathrm{~min}$ at $37^{\circ} \mathrm{C}$. Finally, $2 \mathrm{~mol} / \mathrm{L} \mathrm{H}_{2} \mathrm{SO}_{4}$ was added and the plates were read at $450 \mathrm{~nm}$ using a Benchmark microplate reader (BIORAD, Hercules, CA, USA).

\section{In vivo and in vitro NIR Imaging}

In vivo NIR imaging was performed using a self-built NIR imaging system. This NIR imaging system has been introduced in detail in our previous work [18]. In brief, a helium-neon laser $(1=765.9 \mathrm{~nm})$ is defocused to provide a broad spot with even optical density, and another $808 \mathrm{~nm}$ laser is supplied as background light. High sensitivity CCD camera detects the reflected light, endogenously generated luminescence or fluorescence emission. An $800 \mathrm{~nm}$ long pass filter could blocked the laser light (765 nm) efficiently.

Nine tumor-bearing nude mice were randomly divided into two groups. The experimental group (group A, $\mathrm{n}=5$ ) and control group (group $\mathrm{B}, \mathrm{n}=4$ ) were both administrated anti-Sp17-ICG-Der-02 and free ICG-Der-02 through caudal vein injection. The dose for each animal was $5 \mu \mathrm{g}$, calculated as the amount of ICG-Der-02. The subjected mouse was anesthetized in an isoflurane chamber and immobilized in a Lucite jig before wholebody imaging at predetermined intervals $(1 \mathrm{~h}, 2 \mathrm{~h}, 4 \mathrm{~h}$, 6 h, 1 day, 2 days, and 3 days) post-injection. Two animals from the experimental group were observed until $7 \mathrm{~d}$ post-injection. Other animals were killed at 1 day and 3 days post-injection, and the tumor and major organs were taken out for ex vivo optical imaging examinations. All fluorescence images were acquired with $1 \mathrm{~s}$ exposure $(\mathrm{f} /$ stop $=4)$.

\section{Results}

Overexpression of Sp17 in hepatocellular carcinoma cells Through immunocytochemistry and immunohistochemistry, strong positive staining was observed in the human hepatocellular carcinoma cell line SMMC-7721 and its tumor xenografts tissues (Figure 1). We found Sp17 mainly localized on the cell surface of in vitro cultured cells and both surface and cytoplasm of xenografts tissues. This result suggested that Sp17 could be used as a marker for in vivo molecular imaging and targeting therapy.

\section{Characterization of anti-Sp17-ICG-Der-02}

The anti-Sp17 antibody was conjugated with ICG-Der02 for in vivo tracing of the dynamics of anti-Sp17ICG-Der-02 in nude mice subjects. The NHS ester of the NIR fluorescence dyes is reacted with the amino group of the amino acid residue in anti-Sp17 and purified by dialysis. The absorption and fluorescence emission spectra of the complex were characterized, as shown in Figure 2. The antibody activity of anti-Sp17ICG-Der-02 was tested with ELISA, and the result showed that the antibody on the conjugate retained major biological activity compared with naked antibody (Figure 3).

\section{In vivo targeting capability of anti-Sp17-ICG-Der-02}

The in vivo dynamic processes of anti-Sp17-ICG-Der-02 and corresponding blank samples in tumor-bearing nude mice were evaluated with an NIR fluorescence imaging system. For the experimental group, ICG-Der02 had apparent accumulation in tumor sites at $2 \mathrm{~h}$ post-injection. The fluorescence intensity in the region of interest (ROI) was persistently enhanced and reached the maximum at $24 \mathrm{~h}$ post-injection. Strong fluorescence was observed even at 7 days post-injection for mice in this group. Images of group B (the control group) indicated that free ICG-Der-02, without the help of anti-Sp17, had little accumulation in tumor tissue at $24 \mathrm{~h}$ post-injection. The targeting capability of antiSp17-ICG-Der-02 for tumors was observed both in vivo imaging and ex vitro imaging (Figure 4 and Figure 5) after the process of entrapment. ICG-Der-02 accumulated in the liver then cleared through urine, so the liver 


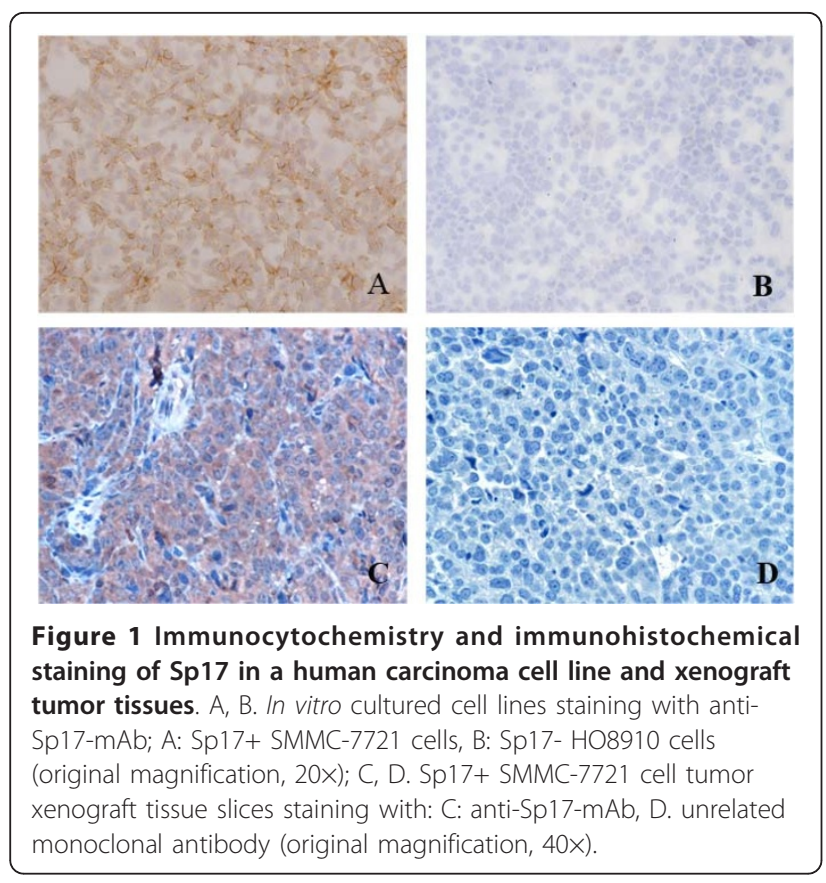

and kidneys showed the strongest fluorescence after injection but the intensity tapered with time. From our results, we know that free ICG-Der-02 was excreted faster than anti-Sp17-ICG-Der-02.

\section{Discussion}

Hepatocellular carcinoma (HCC) is a challenging malignancy of global importance. It is associated with a high rate of mortality and its prevalence in the United States and Western Europe and in China is increasing [19]. Early noninvasive diagnosis is needed for interventional therapy, surgery and reviewing curative effect.

Currently, the requirements for a cell surface molecule and its ligand (antibody) to be suitable as molecular imaging and targeted therapy are stringent. It is highly

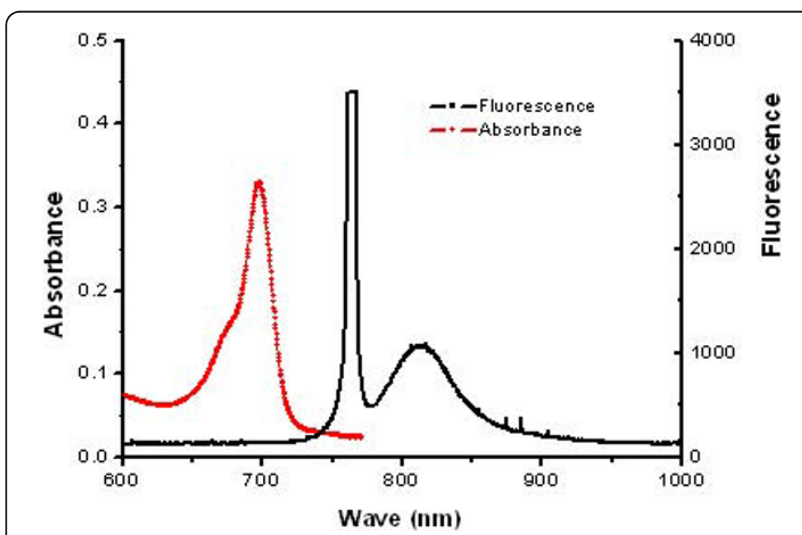

Figure 2 Optical characterization of ICG-Der-02-labled antiSp17.

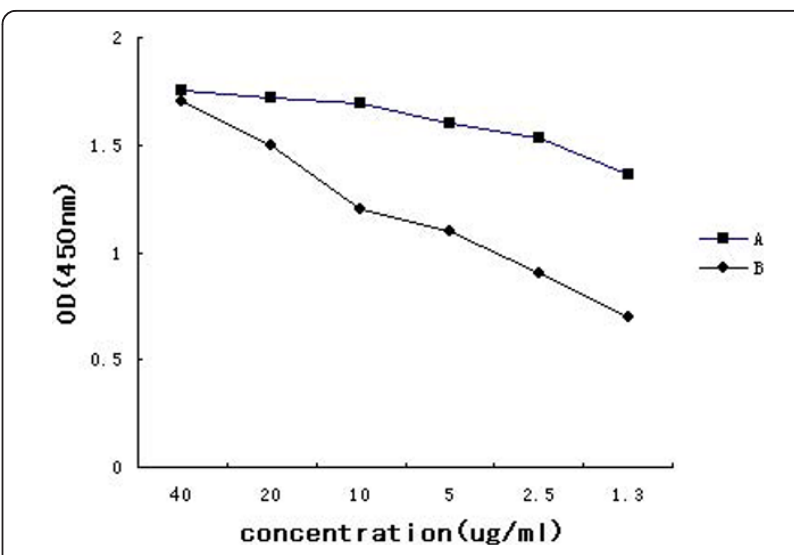

Figure 3 The antibody activity of anti-Sp17-ICG-Der-02 tested with ELISA. A. naked anti-Sp17 antibody; B. anti-Sp17-ICG-Der-02 conjugate.

desirable to find an antibody that can be used to crosslink "probe molecules" for biomarker-targeted specific binding, which can not only provide sensitive and specific imaging information in cancer patients but can also selectively deliver anticancer drugs to tumor sites.

Sp17-expressing SMMC-7721 cells were selectively detected in our study with a whole-body small-animal NIR imaging system to prospectively determine the targeting activity of anti-Sp17 monoclonal antibody. Sp17 was identified as a novel cancer-testis antigen, with overexpression in various malignancies and a low level of expression in some normal tissues (including liver) [20]. We found that Sp17 was overexpressed on the surface of the hepatocellular carcinoma cell line SMMC7721 and retained a high level of expression in xenografts in mice; thus it could be used as a suitable marker for hepatocellular carcinoma. Sp17 is a highly immunogenic protein; more than $90 \%$ of vasectomized males develop immunity against Sp17 without any harm, suggesting that $\mathrm{Sp} 17$ is safe for specific antibody-armed diagnosis and therapy.

The potential use of the high-affinity probe anti-Sp17 for specific NIR imaging in in vivo tumor diagnosis may have advantages over the existing techniques for early diagnosis of tumors. It is a noninvasive technique for in vivo real-time monitoring or tracing of biological information and signals in living subjects [21,22]. In this study, anti-Sp17 antibody-based targeted in vivo NIR imaging was investigated using ICG-Der- 2 as a tracer. In vivo whole-body fluorescence imaging of tumors in mice with anti-Sp17-ICG-Der-02 and free ICG-Der-02 showed that tumors within mice could be clearly differentiated from normal tissues. Particularly, 3 days after application of the high-affinity probe, the most pronounced relative fluorescence signals in the tumors compared with the free dye were observed. The results 

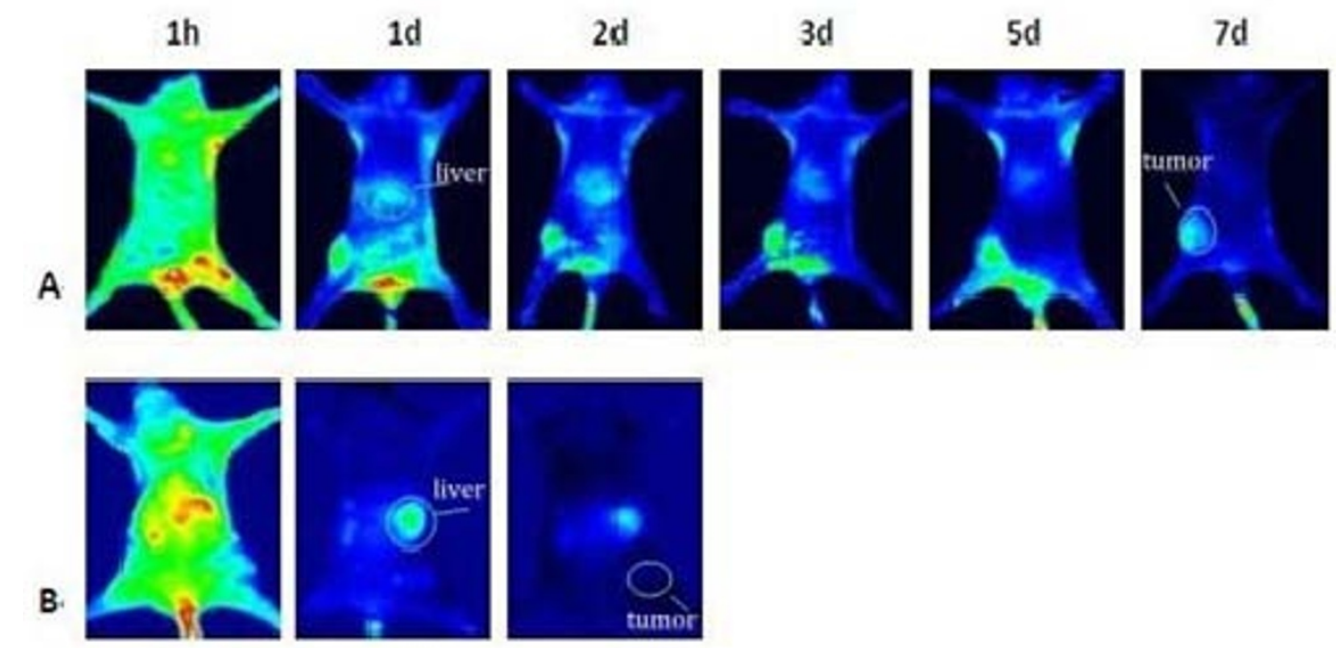

Figure $4 \mathrm{lv}$ vivo images of tumor-bearing mice show the tumor targeting effect of anti-Sp17-ICG-Der-02 (dose for each group was 0.2 $\boldsymbol{\mu g}$, calculated as the amount of ICG-Der-02). A. Systemic injection of anti-Sp17-ICG-Der-02 $(n=5)$. Images were obtained in one mouse; bright fluorescent in the tumor region is due to probe accumulation. B. Systemic injection of free ICG-Der-02 $(n=3)$, images were obtained in one mouse, fluorescent signal in tumor is virtually absent.

showed that anti-Sp17-ICG-Der-02 maintain both the properties of the antibody and photo stability. The antiSp17 mAb revealed excellent targeting effect for tumors in vivo without non-specific binding.

\section{Conclusions}

This in vivo work demonstrates that a new high-affinity antibody identifies the presence of Sp17 expression associated with the site and size of human hepatocellular carcinoma in mice. Anti-Sp17-ICG-Der-02 targeted and accumulated in Sp17 positive tumors in vivo, which

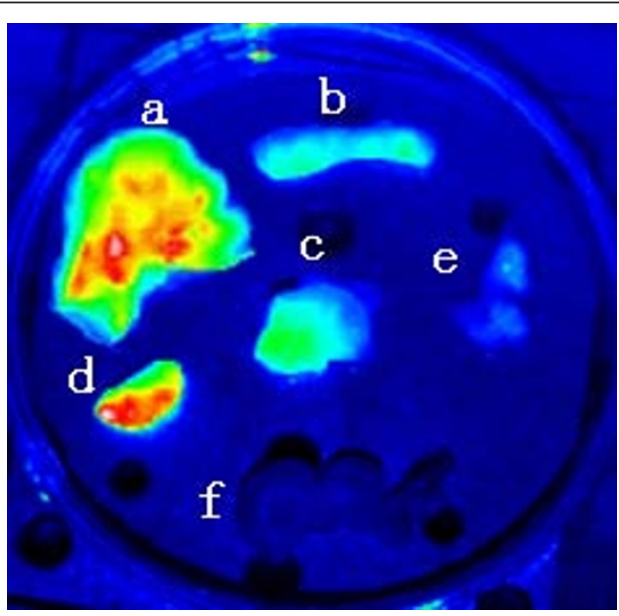

Figure 5 Ex vivo image of tumor and organs from tumorbearing mice with systemic injection of anti-Sp17-ICG-Der-02, 1 day post-injection. The fluorescent intensity from high to low is liver(a), kidney(d), tumor(c), spleen(b), lung(e) and colon(f). demonstrated its capability of serving as a diagnostic reagent.

\section{Abbreviations}

Sp17: Sperm protein 17; NIR: Near-infrared; ICG-Der-02: Indocyanine Green derivative 02; SBR: Signal-to-background; DAB: Diaminobenzidine; EDC: 1-

Ethyl-3-(3-dimethylaminopropyl) carbodiimide; NHS: N-

hydroxysulfosuccinimide sodium salt; TMB: Tetramethylbenzidine; ROI:

Region of interest; PBS: Phosphate-buffered saline; FCS: fetal calf serum.

\section{Author details}

'Laboratory of Molecular Biology, Institute of Medical Laboratory Sciences, Jinling Hospital, School of Medicine, Nanjing University, Nanjing 210002,

China. ${ }^{2}$ Department of Biomedical Engineering, School of Life Science and

Technology, China Pharmaceutical University, Nanjing, 210009, China.

\section{Authors' contributions}

FQL conceived, coordinated and designed the study, and contributed to the acquisition, analysis and interpretation of data and drafted the manuscript. $S X Z$ and XLA performed the experiment and involved in drafting the article. YQG synthesized anti-Sp17-MPAICG-Der-02 and involved in drafting the article. All of the authors have read and approved the final manuscript.

\section{Competing interests}

The authors declare that they have no competing interests.

Received: 10 December 2010 Accepted: 3 March 2011 Published: 3 March 2011

\section{References}

1. Peng XH, Qian X, Mao H, Wang AY, Chen ZG, Nie S, Shin DM: Targeted magnetic iron oxide nanoparticles for tumor imaging and therapy. Int Nanomed 1998, 3:311-321.

2. Jemal A, Siegel R, Ward E, Hao Y, Xu J, Thun MJ: Cancer statistics, 2009. CA Cancer J Clin 2009, 59:225-49.

3. Nie S, Xing Y, Kim GJ, Simons JW: Nanotechnology applications in cancer. Annu Rev Biomed Eng 2007, 9:257-88.

4. Sengupta S, Sasisekharan R: Exploiting nanotechnology to target cancer. Br J Cancer 2007, 96:1315-19. 
5. Toma A, Otsuji E, Kuriu Y, Okamoto K, Ichikawa D, Hagiwara A, Ito H, Nishimura T, Yamagishi H: Monoclonal antibody A7-superparamagnetic iron oxide as contrast agent of MR imaging of rectal carcinoma. $\mathrm{Br} J$ Cancer 2005, 93:131-6.

6. Dancey $\mathrm{G}$, Begent RH, Meyer T: Imaging in targeted delivery of therapy to cancer. Target Oncol 2009, 4:201-17.

7. Yamasaki N, Richardson RT, O'Rand MG: Expression of the rabbit sperm protein Sp17 in COS cells and interaction of recombinant Sp17 with the rabbit zona pellucida. Mol Reprod Dev 1995, 40:48-55.

8. Dong G, Loukinova E, Smith CW, Chen Z, Van Waes C: Genes differentially expressed with malignant transformation and metastatic tumor progression of murine squamous cell carcinoma. I Cell Biochem Suppl 1997, 28-29:90-100.

9. Lim SH, Wang Z, Chiriva-Internati M, Xue Y: Sperm protein 17 is a novel cancer-testis antigen in multiple myeloma. Blood 2001, 97:1508-1510.

10. Straughn JM Jr, Shaw DR, Guerrero A, Bhoola SM, Racelis A, Wang Z, Chiriva-Internati M, Grizzle WE, Alvarez RD, Lim SH, Strong TV: Expression of sperm protein 17 (Sp17) in ovarian cancer. Int J Cancer 2004, 108:805-811.

11. Li FQ, Han YL, Liu Q, Wu B, Huang WB, Zeng SY: Aberrant expression of sperm protein 17 increase migration and chemoresistance of human epithelial ovarian cancer cells. BMC cancer 2009, 9:323.

12. Grizzi F, Gaetani P, Franceschini B, Di leva A, Colombo P, Ceva-Grimaldi G, Bollati A, Frezza EE, Cobos E, Rodriguez y Baena R, Dioguardi N, ChirivaInternati M: Sperm protein 17 is expressed in human nervous system tumours. MBC Cancer 2006, 6:23-29.

13. Gupta G, Sharma R, Chattopadhyay TK, Gupta SD, Ralhan R: Clinical significance of sperm protein 17 expression and immunogenicity in esophageal cancer. Int J Cancer 2007, 120:1739-1747.

14. Li FQ, Liu Q, Han YL, Wu B, Yin HL: Sperm protein 17 is highly expressed in endometrial and cervical cancers. BMC Cancer 2010, 10:429.

15. Kaijzel LEric, van der Pluijm Gabri, Lowik WGMClemens: Whole-body optical imaging in animal models to assess cancer development and progression. Clin Cancer Res 2007, 13:3490-3497.

16. Sampath L, Kwon S, Ke S, Wang W, Schiff R, Mawad ME, Sevick-Muraca EM: Dual-labeled trastuzumab- based imaging agent for the detection of human epidermal growth factor receptor 2 overexpression in breast cancer. J Nucl Med 2007, 48:1501-1510.

17. Qian H, Gu Y, Wang M, Achilefu S: Optimization of the near infrared fluorescence labeling for in vivo monitoring of a protein drug distribution in animal model. J Fluorescence 2009, 19:277-284.

18. Zhang J, Deng D, Qian Z, Liu F, Chen X, An L, Gu Y: The targeting behavior of folate-nanohydrogel evaluated by near infrared imaging system in tumor-bearing mouse model. Pharm Res 2010, 27:46-55.

19. Cabibbo G, Craxì A: Epidemiology, risk factors and surveillance of hepatocellular carcinoma. Eur Rev Med Pharmacol Sci 2010, 14:352-355.

20. Zhang Y, Wang Z, Robinson WR, Lim SH: Combined real time PCR and immunohistochemical evaluation of sperm protein 17 as a cancer-testis antigen. Eur J Haematol 2004, 73:280-284.

21. Ogawa M, Kosaka N, Choyke PL, Kobayashi H: In vivo molecular imaging of cancer with a quenching near-infrared fluorescent probe using conjugates of monoclonal antibodies and indocyanine green. Cancer Res 2009, 69:1268-1272.

22. Lisy MR, Goermar A, Thomas C, Pauli J, Resch-Genger U, Kaiser WA, Hilger I: In vivo near-infrared fluorescence imaging of carcinoembryonic antigenexpressing tumor cells in mice. Radiology 2008, 247:779-787.

doi:10.1186/1756-9966-30-25

Cite this article as: $\mathrm{Li}$ et al:: In vivo Molecular targeting effects of antiSp17- ICG-Der-02 on hepatocellular carcinoma evaluated by an optical imaging system. Journal of Experimental \& Clinical Cancer Research 2011 30:25.

\section{Submit your next manuscript to BioMed Central and take full advantage of:}

- Convenient online submission

- Thorough peer review

- No space constraints or color figure charges

- Immediate publication on acceptance

- Inclusion in PubMed, CAS, Scopus and Google Scholar

- Research which is freely available for redistribution 